\title{
Dynamics of sterol synthesis during development of Leishmania spp. parasites to their virulent form
}

\author{
Chaoqun $\mathrm{Yao}^{1 *}$ and Mary E. Wilson ${ }^{2,3}$
}

\begin{abstract}
Background: The Leishmania spp. protozoa, the causative agents of the "neglected" tropical disease leishmaniasis, are transmitted to mammals by sand fly vectors. Within the sand fly, parasites transform from amastigotes to procyclic promastigotes, followed by development of virulent (metacyclic) promastigote forms. The latter are infectious to mammalian hosts. Biochemical components localized in the parasite plasma membrane such as proteins and sterols play a pivotal role in Leishmania pathogenesis. Leishmania spp. lack the enzymes for cholesterol synthesis, and the dynamics of sterol acquisition and biosynthesis in parasite developmental stages are not understood. We hypothesized that dynamic changes in sterol composition during metacyclogenesis contribute to the virulence of metacyclic promastigotes.

Methods: Sterols were extracted from logarithmic phase or metacyclic promastigotes grown in liquid culture with or without cholesterol, and analyzed qualitatively and quantitatively by gas chromatograph-mass spectrometry (GC-MS). TriTrypDB was searched for identification of genes involved in Leishmania sterol biosynthetic pathways.

Results: In total nine sterols were identified. There were dynamic changes in sterols during promastigote metacyclogenesis. Cholesterol in the culture medium affected sterol composition in different parasite stages. There were qualitative and relative quantitative differences between the sterol content of virulent versus avirulent parasite strains. A tentative sterol biosynthetic pathway in Leishmania spp. promastigotes was identified.

Conclusions: Significant differences in sterol composition were observed between promastigote stages, and between parasites exposed to different extracellular cholesterol in the environment. These data lay the foundation for further investigating the role of sterols in the pathogenesis of Leishmania spp. infections.
\end{abstract}

Keywords: Leishmania, Sterol, Promastigotes, Metacyclogenesis, Virulence, Sterol biosynthetic pathway

\section{Background}

Leishmania spp. are the etiological agents of leishmaniasis, a group of parasitic diseases that are endemic in 88 countries on four continents [1]. Over 20 Leishmania spp. are collectively responsible for varied clinic manifestations of human disease. Three major clinical forms include cutaneous leishmaniasis (CL), mucocutanous leishmaniasis and visceral leishmaniasis (VL). Data compiled by WHO show one million cases of $\mathrm{CL}$ in the last

\footnotetext{
* Correspondence: chyao@rossvet.edu.kn

${ }^{1}$ Department of Biomedical Sciences and One Health Center for Zoonoses and Tropical Veterinary Medicine, Ross University School of Veterinary Medicine, P.O. Box 334, Basseterre, St. Kitts, West Indies

Full list of author information is available at the end of the article
}

five years, and 300,000 cases of VL with 20,000 deaths annually (http://www.who.int/leishmaniasis/en/).

The Leishmania spp. protozoa alternate between a flagellated promastigote in the sand fly vector and an obligate intracellular amastigote, which lacks an external flagellum, in the mammalian host. After a sand fly vector takes a blood meal, amastigotes transform in the sand fly gut to procyclic promastigotes and several other intermediate stages, multiply by binary fission, and eventually develop to the metacyclic promastigotes which are infectious for mammalian hosts. Metacyclic promastigotes are inoculated by the sand fly vector during a blood meal into a new mammalian host [2]. This process of development from the procyclic to the metacyclic promastigotes 
is termed metacyclogenesis. Due to the technical challenges in raising sand flies in laboratory, metacyclogenesis is often modeled in liquid medium by culture of promastigotes in vitro from logarithmic to stationary growth phase, from which the metacyclic promastigotes can be isolated by density for some Leishmania spp. to more than $95 \%$ purity [3]. Metacyclic promastigotes are morphologically distinguishable from procyclic cells by their elongated flagellum (at least double the cell body length), and their smaller body size.

Unlike mammalian cells but similar to fungi, Leishmania spp. are eukaryotic kinetoplastids that synthesize ergosterol [4]. They do not have the enzymes to synthesize cholesterol, although they have detectable cholesterol that they must take up from their external environment [5]. The sterols ergosterol or cholesterol are essential components of plasma membranes found in lipid rafts, membrane microdomains which are also classified as detergent resistant membranes (DRMs) due to their physicochemical properties [6, 7]. Our prior studies using the lipid-raft disrupting agent methyl- $\beta$-cyclodextrin $(M \beta C D)$ suggested that lipid rafts play a pivotal role in the virulence of Leishmania spp. [8]. The anti-fungal agent amphotericin B is widely used in patients with leishmaniasis, especially in regions where parasites are resistant to standard therapy with antimony compounds $[9,10]$, or in patients co-infected with Leishmania sp. and human immunodeficiency virus (HIV) [11]. Amphotericin B preferentially binds to ergosterol, leading to disruption of the osmotic integrity of the membrane in target cells [12].

Given the association between sterol content and heat resistance of $L$. major [13], the objectives of current study were (i) to identify and quantify sterols in distinct stages of Leishmania infantum promastigotes that differ in their virulence for a mammalian host, and (ii) to determine the effects of cholesterol addition or depletion on promastigote sterol (particularly ergosterol) content. The data should provide a baseline for further study of drug- or environmentally-induced changes in parasite sterol content in the pathogenesis and control of Leishmania spp. infections.

\section{Methods}

\section{Ethics Statement}

The Animal Care and Use Committee of the Iowa City Veterans' Affairs Medical Center reviewed and approved protocols for procedures used (Protocols number 1190301 and 1190302). The animal care and use were in accordance with the recommendations in the Guide for the Care and Use of Laboratory Animals of the National Institute of Health.

\section{Parasites}

A Brazilian strain of Leishmania infantum (previously called L. chagasi) (MHOM/BR/00/1669) was serially passaged in golden hamsters to maintain parasite virulence. Amastigotes were isolated from infected hamster spleens and allowed to transform to promastigotes in HOMEM (Hemoflagellate modified Minimal Essential Medium) with $10 \%$ fetal calf serum (FCS) and hemin made from commercially available ingredients as previously described [8]. Serum-free medium (SFM) was prepared similar to HOMEM but lacking FCS and with additional biopterin, adenosine and bovine serum albumin. Virulent promastigotes were used within three passages post-isolation from hamster. An avirulent strain L5 was derived from the same $L$. infantum isolate by serial passage in vitro for over five years as described [14].

Cultures of virulent or avirulent promastigotes were seeded at $1 \times 10^{6}$ cells $/ \mathrm{ml}$ in HOMEM or SFM, and allowed to grow at $26{ }^{\circ} \mathrm{C}$ to the appropriate cell density, monitored daily by microscopic exam on a hemacytometer. Metacyclic promastigotes were isolated from Day 8 stationary-phase cultures of virulent strain parasites on a discontinuous Ficoll gradient as described [3]. Metacyclic promastigotes could not be recovered from similar cultures of avirulent promastigotes (data not shown). In some experiments, metacyclic promastigotes were incubated in $25 \mathrm{mM}$ M $\beta C D$ (Sigma, St. Louis, MO) freshly prepared in RPMI 1640 (GIBCO) at a cell density of $2 \times 10^{8}$ cells $/ \mathrm{ml}$ at room temperature for $1 \mathrm{~h}$ to deplete cellular sterols, and then washed twice by centrifugation in Hanks' balanced salt solution (GIBCO) as described $[8,15]$.

\section{Sterol isolation from promastigotes}

Sterols were extracted from logarithmic phase, stationary phase or metacyclic promastigotes with dicholoromethane and methanol (2:1 ratio [vol/vol]; Sigma) by sequential centrifugation according to a published protocol [16] with some modifications as previously described [8]. Dry sterol aliquots from $2 \times 10^{8}$ promastigote equivalents were maintained at $-20{ }^{\circ} \mathrm{C}$ immediately after extraction until a single use. Extracted sterols were saponified in $30 \% \mathrm{KOH}$ in methanol and derivatized with dichloromethane and bis (trimethylsilyl) trifluroacetamide (BSTFA) (Sigma) in 1:2 ratio following a previously-described protocol [8].

\section{Sterol identification}

Dry derivatized trimethylsilyl derivatives were analyzed in the High Resolution Mass Spectrometry Facility at the University of Iowa using a ThermoFinnigan Voyager single quadruple mass spectrometer interfaced with a Trace2000 gas chromatograph (GC). The original software from the supplier was used for both data acquisition and processing. A DB-5ht capillary column (inner diameter, $0.25 \mathrm{~mm}$; length $30 \mathrm{~m}$ ) (JW Scientific Inc., 
Folsom, CA) was used. The GC temperature program settings were $175{ }^{\circ} \mathrm{C}$ for $1 \mathrm{~min}$, ramp up at $10{ }^{\circ} \mathrm{C} / \mathrm{min}$ to $280{ }^{\circ} \mathrm{C}$, and hold at $280{ }^{\circ} \mathrm{C}$ for $30 \mathrm{~min}$. The $\mathrm{GC}$ inlet temperature was set at $280{ }^{\circ} \mathrm{C}$. The GC eluent was electronically ionized at $70 \mathrm{eV}$. The mass spectrometer was programmed to detect a mass range of 45-700 Da.

Sterol identification was achieved by two independent methods. First, the GC retention time of same chemical compound is very consistent under the same experimental conditions (GC column, solvent, temperature etc.). Therefore, known chromatograph grade cholesterol and ergosterol standards (Sigma) were used every time to generate spectra, used to identify the corresponding sterol in individual samples by comparison. For ergosterol-related compounds lacking appropriate standards, the GC retention time was used once identified (see below). Second, the unique mass spectrum of each GC peak was searched against the small-molecule library (2001 version) of the National Institute of Standards and Technology library (NIST)/EPA/NIH Mass Spectral Library, which was purchased with the GC-MS instrument. The library search program was integrated in the Xcalibur operating software. Positive identification of a sterol was made if its mass spectrum matched a sterol in the library with a SI/RSI matching factor score $\geq 780$ at a probability score of at least 50 .

\section{Sterol quantification}

Two approaches were used to quantify sterols, capable of either relative or absolute quantification. Multiple preliminary trials showed that either cholesterol or ergosterol amounts were proportionally and linearly correlated with the area under its respective sterol peak on GC spectra. Thus the area under peaks was used for relative quantification of sterol components. For each promastigote stage, the total amount of all sterols added up to $100 \%$.

The absolute amount of cholesterol was also quantified as follows. A standard curve was generated for each experiment using varying amounts of chromatographgrade cholesterol that was deuterated at six positions ( $\left[{ }^{2} \mathrm{H}\right]$ cholesterol; Sigma), and varying amounts of chromatograph-grade ergosterol (Sigma). Quality control for each experiment was achieved by comparing multiple standard curves run at the time of each experiment, requiring a correlation factor of 0.98 or higher between standard amounts and retention times/area under curves. Samples and standards that did not meet quality control were rerun. Known amounts of $\left[{ }^{2} \mathrm{H}\right]$ cholesterol were also spiked into each sample, and samples were analyzed at three levels of cell equivalents to make sure $\left[{ }^{2} \mathrm{H}\right]$ cholesterol from at least two cell equivalents were within the range of the standard curve. The absolute amount of unknown cholesterol in each sample was determined by its area relative to that of $\left[{ }^{2} \mathrm{H}\right]$ cholesterol standards. Amounts of ergosterol and other ergosterolrelated sterols for which deuterated standards were not available were calculated considering their relative abundance compared to the absolute amount of cholesterol in the same sample. The peak areas under curves were used to generate the extrapolated values.

\section{Sterol biosynthetic pathway (SBP) of Leishmania spp.}

SBP genes of Trypanosoma cruzi have been identified in a recent publication [17]. Locus identifiers of T. cruzi SBP genes were used as gene ID to search TriTryp database (http://tritrypdb.org/tritrypdb/, latest access date 01/30/ 16), resulting in identification $L$. major homologues, which were used to pull out all Leishmania spp. genes. In case of failure, keywords of the current annotation of SBP genes of $T$. cruzi were used as gene text during search. Ergosta7,22-dien-3 $\beta$-ol was a unique case; keyword "ergosta" was used to search Kyoto Encyclopedia of Genes and Genomes (KEGG) website (http://www.genome.jp/kegg/, latest access date 01/30/16) to find KEGG reactions and enzymes. Afterwards, keyword "hydroxysteroid" was used to identify genes in TriTryp database. Three genes were identified in L. infantum, one of which was a conserved hypothetic protein. The protein sequence was used to BLAST and a putative hydroxy steroid dehydrogenase/isomerase was identified. Accession numbers for individual genes were identified by BLAST search.

\section{Results}

\section{Sterol identification}

We previously identified five sterols species from infectious $L$. infantum metacyclic promastigotes cultured in complete HOMEM in vitro [8]. The current investigations included logarithmic, stationary and metacyclic stage promastigotes in virulent (recent isolate) and avirulent L5 strains maintained in either HOMEM or SFM. All five of the previously identified sterols (cholesterol, two isomers of ergosterol with distinct GC retention time, ergosta-7,22-dien-3 $\beta$-ol, and stigmasta-7,24(28)-dien-3 $\beta$-ol) were identified in addition to four new sterols (ergostatetraenol, an additional isomer of ergosta-7,22-dien-3 $\beta$-ol, zymosterol and lanosterol; Table 1; Fig. 1).

\section{Dynamic changes in sterols during promastigote metacyclogenesis}

Discovery of sterols that are differentially represented in metacyclic versus less virulent promastigotes may provide clues to the differentiation and pathogenicity of the metacyclic promastigotes. Therefore sterols were isolated from a recent isolate of virulent L. infantum promastigotes cultured in HOMEM with 10 \% FCS in vitro, in either logarithmic (day 3 to 4 of culture) or metacyclic 
Table 1 Sterols identified by gas chromatograph-mass spectrometry (GC-MS) in promastigotes of Leishmania infantum derived from in vitro culture

\begin{tabular}{|c|c|c|}
\hline Sterol & Identification $^{a}$ & $R R_{t}^{b}$ \\
\hline Cholesterol & I & 1.000 \\
\hline Zymosterol & New & 1.030 \\
\hline Ergosterol - I & $\|$ & 1.069 \\
\hline Ergosta-7,22-dien-3ß-ol - I & New & 1.078 \\
\hline Ergostatetraenol & New & 1.086 \\
\hline Ergosterol - ॥ & III & 1.115 \\
\hline Ergosta-7,22-dien-3ß-ol - II & $\mathrm{Vl}$ & 1.131 \\
\hline Lanosterol & New & 1.157 \\
\hline Stigmasta-7,24(28)-dien-3ß-ol & $\mathrm{V}$ & 1.229 \\
\hline
\end{tabular}

${ }^{\mathrm{a}} \mathrm{V} \mathrm{V}$ were identified previously [8]. New indicates sterols identified in the current study

${ }^{\mathrm{b}}$ Relative retention time (RRt) to cholesterol on GC, which is the ratio of retention time of each sterol to that of cholesterol

stage. Metacyclic promastigotes were isolated from day 8 stationary-phase promastigotes.

The dominant sterols in logarithmic-phase promastigotes were ergosterols. Ergosterol-I and ergosterol-II, two isomers with distinct retention times on the GC column, accounted for $30.92 \%$ and $39.13 \%$ of total sterols, respectively (Fig. 2a). The third most abundant sterol was cholesterol, accounting for $14.60 \%$ of total sterols. In contrast, in stationary-phase promastigotes the two ergosterol isomers accounted for only $19.09 \%$ and $21.74 \%$, respectively of total sterols, whereas cholesterol was the most abundant and constituted $27.77 \%$ of total sterols (Fig. 2a). Metacyclic promastigotes were different from both; the two ergosterols accounted for only $8.94 \%$ and $5.18 \%$, respectively, of total metacyclic promastigotes sterols. The two most abundant sterols in metacyclics were ergosta-7,22-dien-3 $\beta$-ol-II and cholesterol, accounting for $37.90 \%$ and $29.11 \%$ of total sterols, respectively. The differences in sterols among the various promastigote stages were statistically significant (Fig. 2a), suggesting a stage-specific expression of various sterols in virulent $L$. infantum promastigotes.

We also analyzed sterols of a L. infantum avirulent L5 strain, which was derived by continuous culturing in vitro of a wild-type virulent strain for over 5 years as previously described [14]. As previously shown L5 appeared to undergo no metacyclogenesis, since almost no metacyclic promastigotes were recovered from stationary-phase L5 promastigotes $(<0.1 \%$ recovery rate, compared to $13.4 \%$ in the wild-type strain) [3]. In this strain, the most abundant sterols in both log and stationary phase promastigotes were ergosterol-II, accounting for $39.93 \%$ and $39.28 \%$ of total sterols, respectively (Fig. 2b). In log phase promastigotes the next two most abundant sterols were ergosterol-I (28.68 \%) and cholesterol (16.68 \%). In stationary cells, they were cholesterol (33.69 \%) and ergosterol-I (18.99 \%) (Fig. 2b). The difference between these two stages were statistically significant (Fig. 2b), indicating there is stage-specific variation in sterols in avirulent $L$. infantum, even in the absence of the capacity to undergo metacyclogenesis.

\section{Cholesterol in the culture medium affected sterol composition in L. infantum promastigotes}

Trypanosomatid protozoa do not have the enzymes to synthesize cholesterol, and it has been shown that the bloodstream form of $T$. brucei can take up cholesterol from its environment [4]. As such, we hypothesized that the Leishmania culture medium might be an important determinant of the protozoan sterol composition. Promastigotes were cultured in parallel in either HOMEM supplemented with $10 \%$ FCS, thus bathing the parasites in an exogenous source of cholesterol, or in a defined SFM that was similar to HOMEM but lacked serum (and thus lacked cholesterol). Sterols from stationary growth-phase promastigotes incubated in these media were compared. In virulent strain $L$. infantum, cholesterol accounted for $27.77 \%$ and $3.69 \%$ of total sterols in parasites grown in the serum-containing or serum-free media, respectively $(P<0.001$, Fig. $2 \mathrm{c})$. The proportions were $33.69 \%$ and $1.14 \%$ in avirulent L5 strain L. infantum, respectively $(P<0.0001$, Fig. $2 \mathrm{~b})$. These data verified that cholesterol was reduced to minimal amounts in promastigotes incubated in a cholesterol-low environment, while other compounds were not as dramatically altered. Whether these were compensatory changes or merely changes in proportions required us to study the absolute cholesterol amounts, below.

\section{Sterols in virulent versus avirulent strain of $L$. infantum}

Qualitative and quantitative differences in sterol composition between virulent and avirulent Leishmania spp. have not been reported, nor has it been determined whether virulence affects the ability of promastigotes to adsorb cholesterol from their environment. We therefore compared the relative amounts of each sterol component in $\log$ or stationary phase virulent or avirulent strains of L. infantum, cultured in either cholesterolcontaining HOMEM or in cholesterol-free SFM. The results are summarized in Table 2. Several differences were observed. First, both virulent and avirulent $L$. infantum were able to adsorb cholesterol from the environment. Second, the amount of ergosterol-I in virulent $L$. infantum grown in SFM was proportionally greater than the avirulent L5 strain, with the difference made up by a relative decrease in ergosterol-II. Ergosterol-II was also decreased in virulent stationary parasites incubated in HOMEM, but in this case it was compensated by a significant increase in ergosta-7,22-dien-3 $\beta$-ol compared to 


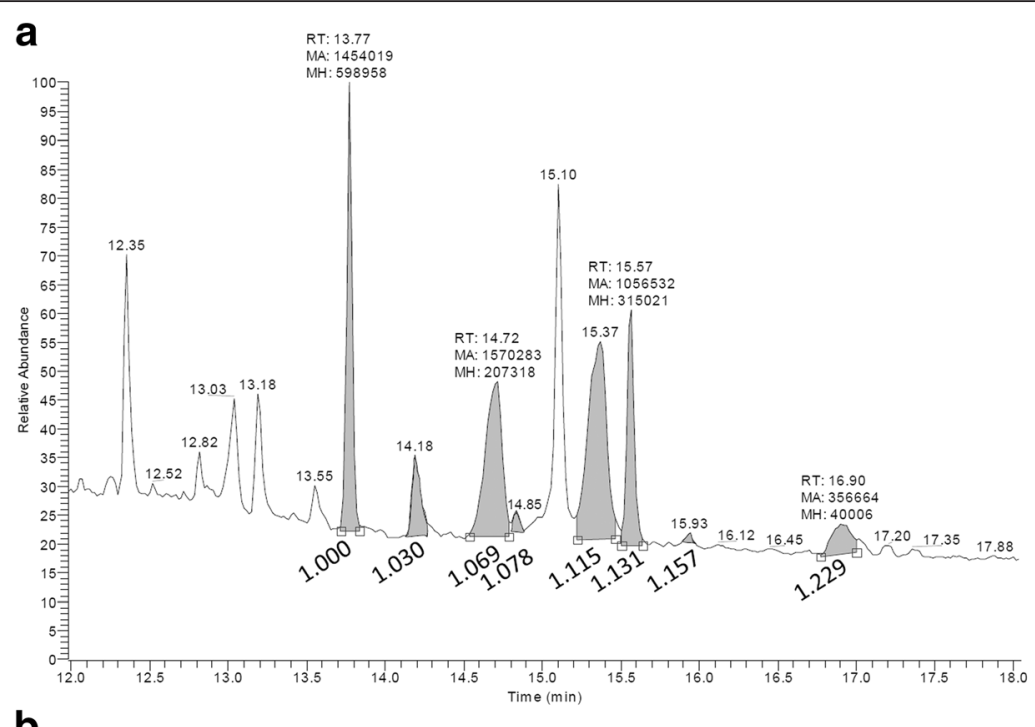

b
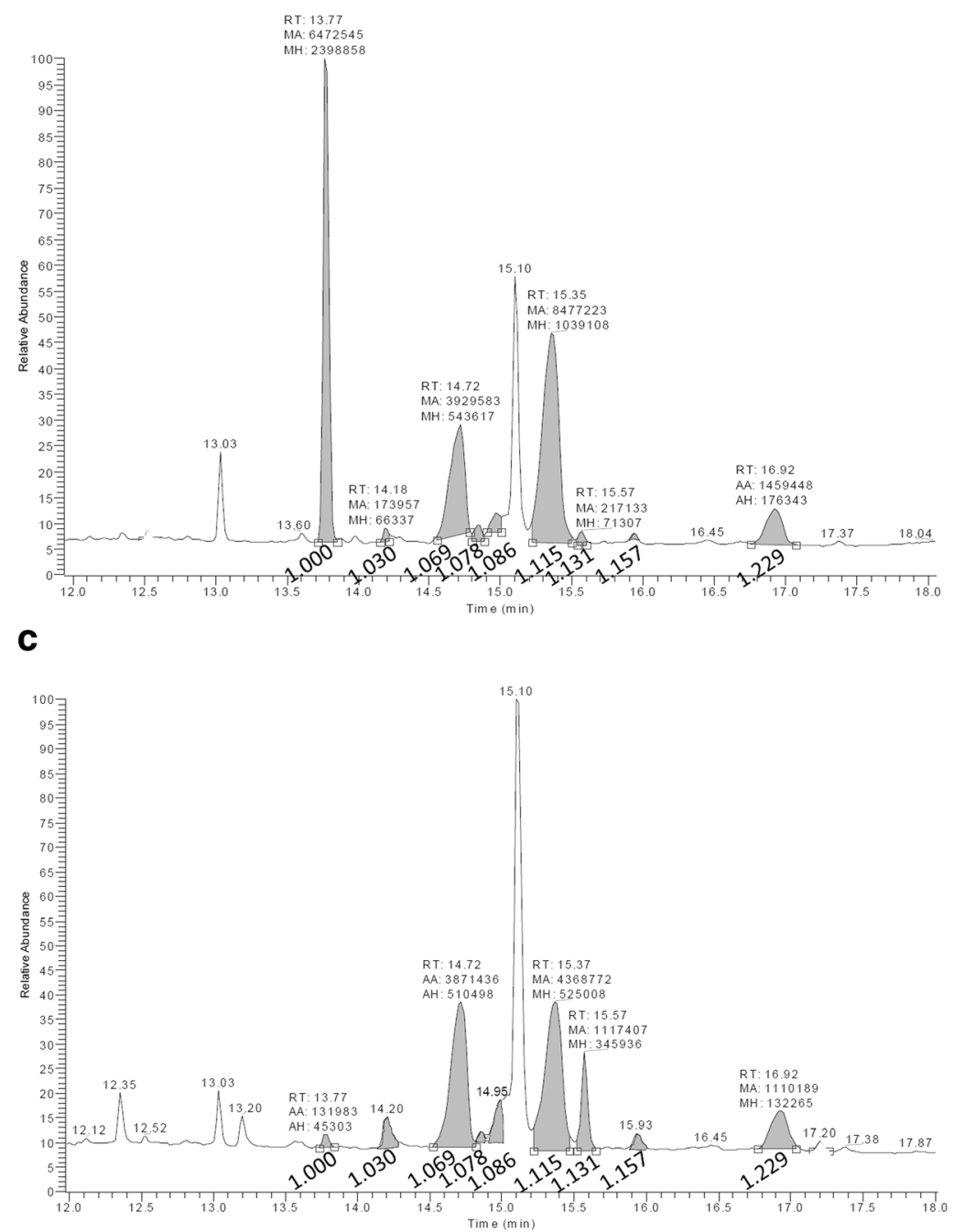

Fig. 1 (See legend on next page.) 
(See figure on previous page.)

Fig. 1 Representative gas chromatography spectra (GC) of total sterols of Leishmania infantum virulent and avirulent strains cultured in complete HOMEM or serum-free medium (SFM). Shaded peaks were identified as sterols with relative retention time to cholesterol as marked. 1.000-Cholesterol;

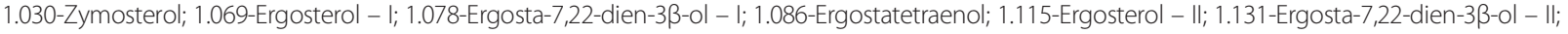
1.157-Lanosterol; and 1.229-Stigmasta-7,24(28)-dien-3ß-ol. a Stationary promastigotes of virulent strain cultured in HOMEM. b Stationary promastigotes of avirulent L5 strain cultured in HOMEM. c Stationary promastigotes of virulent strain cultured in SFM. The GC spectra of metacyclic promastigotes had been previously published [8]

the avirulent L5 strain in either log or stationary growth phase (Table 2). These data demonstrate a difference in sterol synthesis between virulent and avirulent strains that extends beyond the ability to adsorb cholesterol from the external environment.

\section{Dynamic changes in absolute amounts of sterols during promastigote metacyclogenesis}

The above proportional differences in sterol content cannot reveal whether some parasite forms are relatively depleted of membrane sterols. We therefore measured absolute sterol amounts either directly or indirectly as described above. Surprisingly, stationary-phase promastigotes cultured in HOMEM had roughly two times the total sterol content compared to purified metacyclic promastigotes. The most dramatic differences were in cholesterol, and ergosterol-I and -II (Table 3). Depletion of sterols in the metacyclic promastigotes by $\mathrm{M} \beta \mathrm{CD}$ treatment validated the measurements of sterols in other forms.

\section{Absolute sterol content in avirulent or virulent parasites in different culture media}

Absolute quantification revealed that total sterols were higher in either virulent or avirulent L. infantum strains when cultured in a cholesterol-containing medium (HOMEM) than in medium without cholesterol (SFM). Furthermore, virulent parasites contained more total sterols than the avirulent L5 strain grown in either medium. In virulent strain $L$. infantum promastigotes, the amount of cholesterol in stationary phase promastigotes was about 25 times higher when cultured in HOMEM (448.3 ng) than in SFM (18.0 ng). Nevertheless the cells in SFM synthesized more ergosterol-I and -II, i.e., $1001.8 \mathrm{ng}$ in SFM compared to $734.6 \mathrm{ng}$ in the HOMEM (Additional file 1: Table S1). In avirulent L5 strain L. infantum, the cholesterol amount was also many-fold higher when promastigotes were cultured in HOMEM than in SFM. Interestingly and in contrast to the virulent strain, L5 promastigotes in SFM appeared not to have synthesized more ergosterols despite cholesterol depletion (Additional file 1: Table S1).

Leishmania spp. sterol biosynthetic pathway (SBP) Based on the Trypanosoma cruzi genome, homologous SBP genes of $L$. infantum were identified for the multiple reactions from farnesyl diphosphate to ergosterol. Furthermore, an enzyme was identified for the reaction between ergosterol and ergosta-7,22-dien-3 $\beta$-ol. Consequently all identified sterols reported in the current study were fitted in an SBP as shown in Fig. 3. One exception was stigmasta-7,24(28)-dien-3 $\beta$-ol. No entry was found in either the TriTryp or the KEGG databases using keyword "stigmasta". Corresponding genes and accession numbers of other Leishmania spp., including but are not limited to L. donovani, L. major, L. braziliensis, L. mexicana and L. panamensis, were also identified (Additional file 2: Table S2).

\section{Discussion}

The genomes of the trypanosomatid protozoans, including Trypanosoma spp. and Leishmania spp., have genes encoding enzymes that catalyze the biosynthesis of ergosterol but not cholesterol [18-21]. This characteristic is more similar to fungi than other eukaryotes including mammals [22]. Consequently, amphotericin B, which selectively binds to ergosterol leading to disruption of the osmotic integrity of the membrane in target cells [12], is a powerful agent used for treatment of leishmaniasis and fungal infections of medical and veterinary importance. Amphotericin B is widely used as a first-line antileishmanial agent in patients from India where antimony-resistant strains of Leishmania spp. are the rule, or in the case of HIV - Leishmania spp. coinfections [10, 23].

Goad and colleagues (1984) identified major sterols of promastigotes of L. tropica, L. donovani, L. mexicana and L. major by GC-MS[5], and $\mathrm{Xu}$ and colleagues recently quantified sterols of L. major [13]. In keeping with the efficacy of treatment modalities, transient treatment with miltefosine, the first orally active anti-leishmanial drug effective in VL, significantly affects sterol content of $L$. donovani promastigotes. In one study, cholesterol and cholesta-5,7,24-trien-3 $\beta$-ol were $2 \times$ and $32 \times$ higher, respectively, whereas ergosta-7,24(28)-dien-3 $\beta$-ol and ergosta-5,7,24(28)-trien-3 $\beta$-ol were $15 \times$ and $5 \times$ lower, respectively, in miltefosine treated than untreated promastigotes [24]. Similarly, other anti-leishmanial agents such as chalcone and azasterols changed sterol profiles in L. amazonensis $[25,26]$. Drug resistance is associated with significantly altered sterol profiles (e.g. atovaquone-resistant $L$. infantum [16], miltefosine-resistant L. donovani [27]). 
a

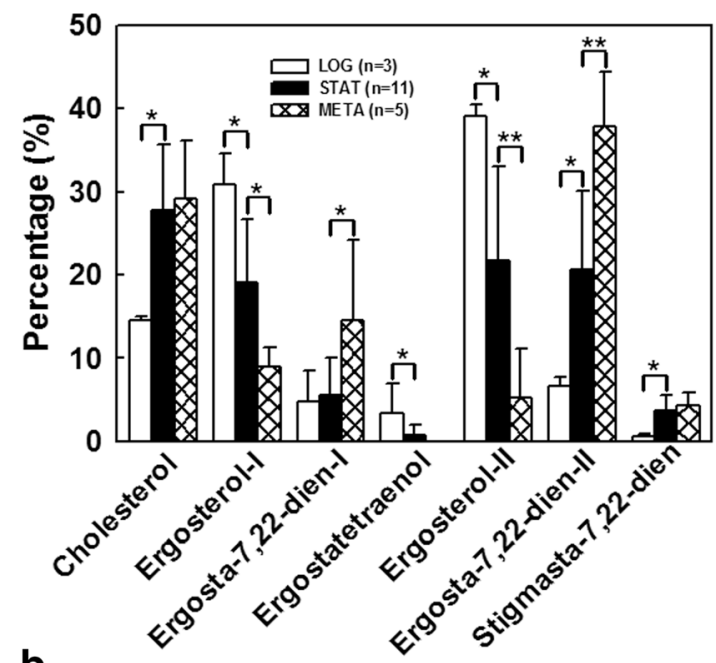

b

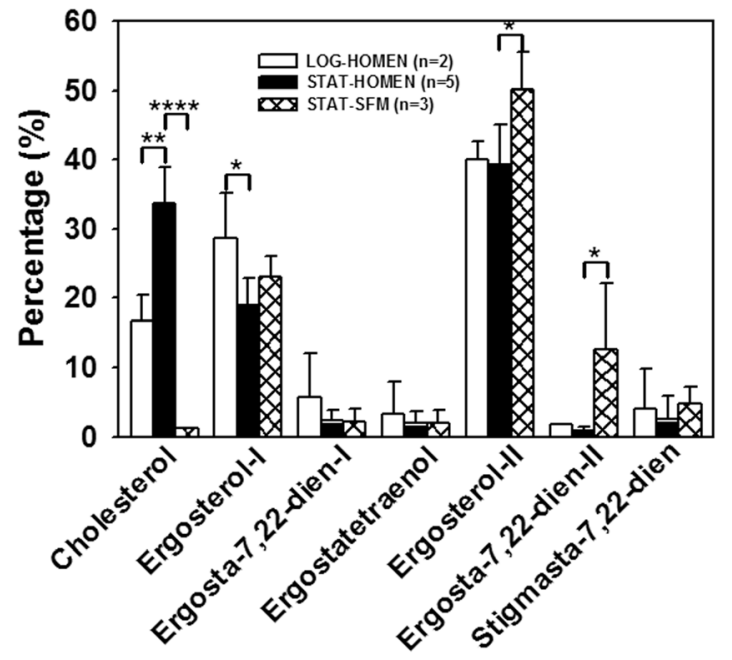

C

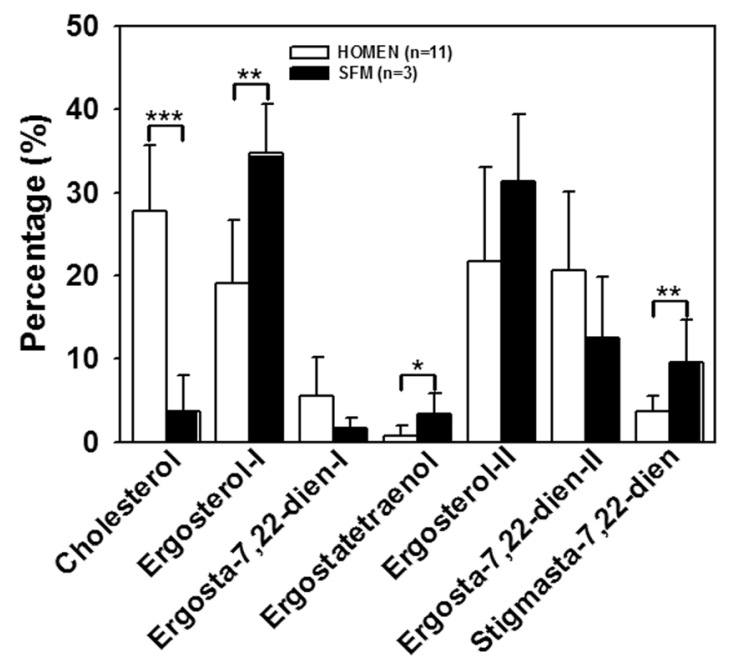

Fig. 2 Sterol amount in percentage (\%) among logarithmic, stationary and metacyclic promastigotes of $L$. infantum virulent or avirulent $L 5$ strain grown in complete HOMEM or serum-free medium (SFM). LOG: logarithmic phase promastigotes; STAT: stationary phase promastigotes; META: metacyclic promastigotes. For each developmental stage, all sterols were added up to $100 \%$. a Virulent strain grown in HOMEM. b Avirulent L5 strain grown in HOMEM or SFM. c Stationary phase promastigotes of virulent strain cultured in HOMEM or SFM. ${ }^{*} P<0.05$; ${ }^{* *} P<0.01 ;{ }^{* * *} P<0.001$; ${ }^{* * * *} P<0.0001$

Despite their inability to synthesize the compound, Trypanosomatids have ample cholesterol when measured [28], leading to questions about the relative importance of different sterols in pathogenesis. Herein we describe qualitative and quantitative analyses of sterol content in L. infantum promastigotes, the causative agent of VL. Our data suggest there is substantial adsorption of cholesterol from the parasite's external environment, complicating interpretation of sterol content. To the best of our knowledge, this is the first report of the absolute amounts of sterols in parasites that differ in virulence and in developmental stage. We also make the unique observation that the sterol content is dramatically altered in different environmental media.

Sterols are identified by their relative retention time (RRt) observing their mass spectrum peak on gas chromatography. Goad and colleagues (1984) measured sterols in logarithmic-phase promastigotes of several Leishmania spp., reporting only trace amounts of cholesterol in cells cultured in lipid-free medium [5]. Ergosta-5,7,24(28)-trien-3 $\beta$-ol was the dominant sterol among various species of Leishmania spp., ranging from about $60 \%$ in L. major, $70 \%$ in L. tropica, L. mexicana and $L$. donovani and up to $90 \%$ in L. amazonensis and L. pifanoi [5]. Consistent with that report, we observed that cholesterol comprised less than $4 \%$ of total sterols in promastigotes cultured in cholesterol-free SFM, with a much higher representation of ergosterol. This was in contrast to much higher percentages of cholesterol in promastigotes cultured in HOMEM containing FCS as a cholesterol source, confirming the ability of parasites in either growth stage to adsorb cholesterol from their external medium. In addition we detected eight sterols other than cholesterol, all presumptively precursors or derivatives of ergosterol (Fig. 3) (discussed below). These observations are consistent with the conclusion that ergosterol is the major sterol synthesized by Leishmania spp. with or without cholesterol in the external environment.

Less abundant sterols included ergosta-7,22-dien-3 $\beta$-ol (20.7\%), stigmasta-7-24(28)-dien-3 $\beta$-ol, ergostatetraenol, zymosterol and lanosterol, all less than $4 \%$. The last three had been found to be precursors of ergosterol in Leishmania spp., T. brucei, T. cruzi and the yeast 
Table 2 Relative sterol content of a recently isolated virulent (vir) strain compared to an avirulent (L5) strain of Leishmania infantum

\begin{tabular}{|c|c|c|c|c|c|c|}
\hline Parasite-growth phase & L5-LOG $(n=2)$ & Vir-LOG $(n=3)$ & L5-STAT $(n=5)$ & Vir-STAT $(n=11)$ & L5-STAT $(n=3)$ & Vir-STAT $(n=3)$ \\
\hline Growth Medium & HOMEM & & & & SFM & \\
\hline \multicolumn{7}{|l|}{ Sterol: } \\
\hline Cholesterol & $16.68(3.70)$ & $14.60(0.38)$ & $33.69(5.25)$ & $27.77(7.94)$ & $1.14(0.13)$ & $3.69(4.28)$ \\
\hline Zymosterol & $0.00(0.00)$ & $0.00(0.00)$ & $0.27(0.38)$ & $0.71(2.17)$ & $0.72(0.63)$ & $2.52(2.25)$ \\
\hline Ergosterol -I & $28.68(6.48)$ & $30.92(3.73)$ & $18.99(3.85)$ & $19.09(7.59)$ & $22.96(3.09)$ & $34.70^{*}(5.98)$ \\
\hline Ergosta-7,22-dien-3ß-ol - I & $5.62(6.48)$ & $4.76(3.74)$ & $2.29(1.58)$ & $5.50(4.64)$ & $2.16(1.94)$ & $1.64(1.33)$ \\
\hline Ergostatetraenol & $3.27(4.62)$ & $3.38(3.57)$ & $1.92(1.72)$ & $0.72(1.25)$ & $1.97(1.80)$ & $3.39(2.39)$ \\
\hline Ergosterol -II & $39.93(2.62)$ & $39.13(1.37)$ & $39.28(5.81)$ & $21.74^{* *}(11.33)$ & $50.17(5.33)$ & $31.33^{*}(8.09)$ \\
\hline Ergosta-7,22-dien-3ß-ol- ॥ & $1.71(0.04)$ & $6.58^{*}(1.20)$ & $0.84(0.66)$ & $20.66^{* * *}(9.43)$ & $12.69(9.50)$ & $12.56(7.28)$ \\
\hline Lanosterol & $0.00(0.00)$ & $0.00(0.00)$ & $0.16(0.36)$ & $0.15(0.35)$ & $1.14(1.07)$ & $0.58(1.00)$ \\
\hline Stigmasta-7,24(28)-dien-3ß-ol & $4.10(5.80)$ & $0.63(0.32)$ & $2.50(3.44)$ & $3.65(1.88)$ & $4.77(2.50)$ & $9.60(5.17)$ \\
\hline
\end{tabular}

Data show the average (standard deviation) percentages of each sterol relative to the total amount of sterols in each cell type, set at $100 \%$. Sterols were identified and quantified by gas chromatograph-mass spectrometry. L. infantum virulent (vir) or avirulent L5 promastigotes were cultured in either HOMEM or serum free medium (SFM) and harvested for sterol analyses in logarithmic (LOG) or stationary (STAT) growth phases

${ }^{*} P<0.05 ;{ }^{* *} P<0.01 ; * * * P<0.001$

Saccharomyces cerevisiae $[4,17,29,30]$. Ergosta-7,22dien-3 $\beta$-ol has been previously detected in $L$. donovani and $L$. amazonensis $[24,26,27]$ as well as in S. cerevisiae yeast and Ganoderma pfeifferi fungus [31, 32]. How it fits to the SBP has not been established. We identified an enzyme, hydroxysteroid dehydrogenase/isomerase (Accession number: XP_001464825.1) in the Leishmania genome, which could potentially catalyze a reaction linking ergosta-7,22-dien-3 $\beta$-ol to ergosterol (Fig. 3). Stigmasta-7-24(28)-dien-3 $\beta$-ol has been found in the fungus Gymnosporangium juniper [33]; ergostatetraenol in Candida albicans and S. cerevisiae [34, 35]; ianosterol in the woody plant pathogen Eutypa lata [36]; and zymosterol in S. cerevisiae [35].

Further discussion of stigmasta-7-24(28)-dien-3 $\beta$-ol is warranted. This sterol has not been found in T. brucei [29] although it is often found in fungi and plants. This observation might have several explanations. First, the validity of stigmasta-7-24(28)-dien-3 $\beta$-ol identification must be considered. The GC-MS peaks favorably argues for its identification, which is shown in the Additional file 3: Fig. S1. Secondly, this sterol might be taken up by Leishmania spp. promastigote from FCS, where it could be present in trace amounts. Our data do not support such a scenario. As showed in Table 2 promastigotes cultured in SFM had higher levels of this sterol compared to the cells derived from HOMEM. Thirdly, biosynthetic enzymes for this sterol might be unique to Leishmania spp. Stigmastanol is formed from either $\beta$ sitosterol by reduction, or through hydrogenation of stigmasterol.

Stigmasterol and sitosterol are phytosterols derived from mevalonate biosynthetic pathways. Isoprenoid synthesis is controlled by 3 -hydroxy-3-methylglutaryl-

Table 3 Absolute amounts of sterols of stationary and metacyclic promastigotes of a virulent strain of Leishmania infantum, incubated in the absence or presence of $M \beta C D^{a}$

\begin{tabular}{|c|c|c|c|}
\hline Sterol & Stationary $(n=6)$ & Metacyclic $(n=6)$ & MßCD-metacyclic $(n=6)$ \\
\hline Cholesterol & $448.3(249.2)$ & $248.9(120.8)$ & $89.0(40.6)^{*}$ \\
\hline Ergosterol -I & $331.0(290.5)$ & $68.4(13.1)$ & $40.3(22.3)^{*}$ \\
\hline Zymosterol & $44.2(108.2)$ & $0.0(0.0)$ & $0.0(0.0)$ \\
\hline Ergosta-7,22-dien-3ß-ol - I & $81.8(40.6)$ & $100.6(45.8)$ & $49.3(17.2)^{*}$ \\
\hline Ergostatetraenol & $0.0(0.0)$ & $0.0(0.0)$ & $0.0(0.0)$ \\
\hline Ergosterol - II & $403.6(437.7)$ & $35.7(33.4)$ & $11.5(9.3)$ \\
\hline Ergosta-7,22-dien-3ß-ol - II & $387.8(282.1)$ & $318.9(137.8)$ & $81.8(37.3)^{* *}$ \\
\hline Lanosterol & $6.4(15.7)$ & $0.0(0.0)$ & $0.0(0.0)$ \\
\hline Stigmasta-7,24(28)-dien-3ß-ol & $62.8(63.6)$ & $37.8(19.4)$ & $17.5(8.6)^{*}$ \\
\hline Total & $1766.0(1307.8)$ & $810.2(239.2)$ & $289.5(120.7)^{* *}$ \\
\hline
\end{tabular}

${ }^{a}$ Numbers indicate the mean (SD) absolute concentrations of sterols ( $\mathrm{ng} / 10^{7}$ cells). Metacyclic promastigotes were isolated from stationary phase promastigotes as previously described [3]. An aliquot of the metacyclic cells were treated with $25 \mathrm{mM} \mathrm{M \beta CD}$

${ }^{*} P<0.05$; ${ }^{* *} P<0.01$ between metacyclic and $M \beta C D$-treated metacyclic promastigotes 


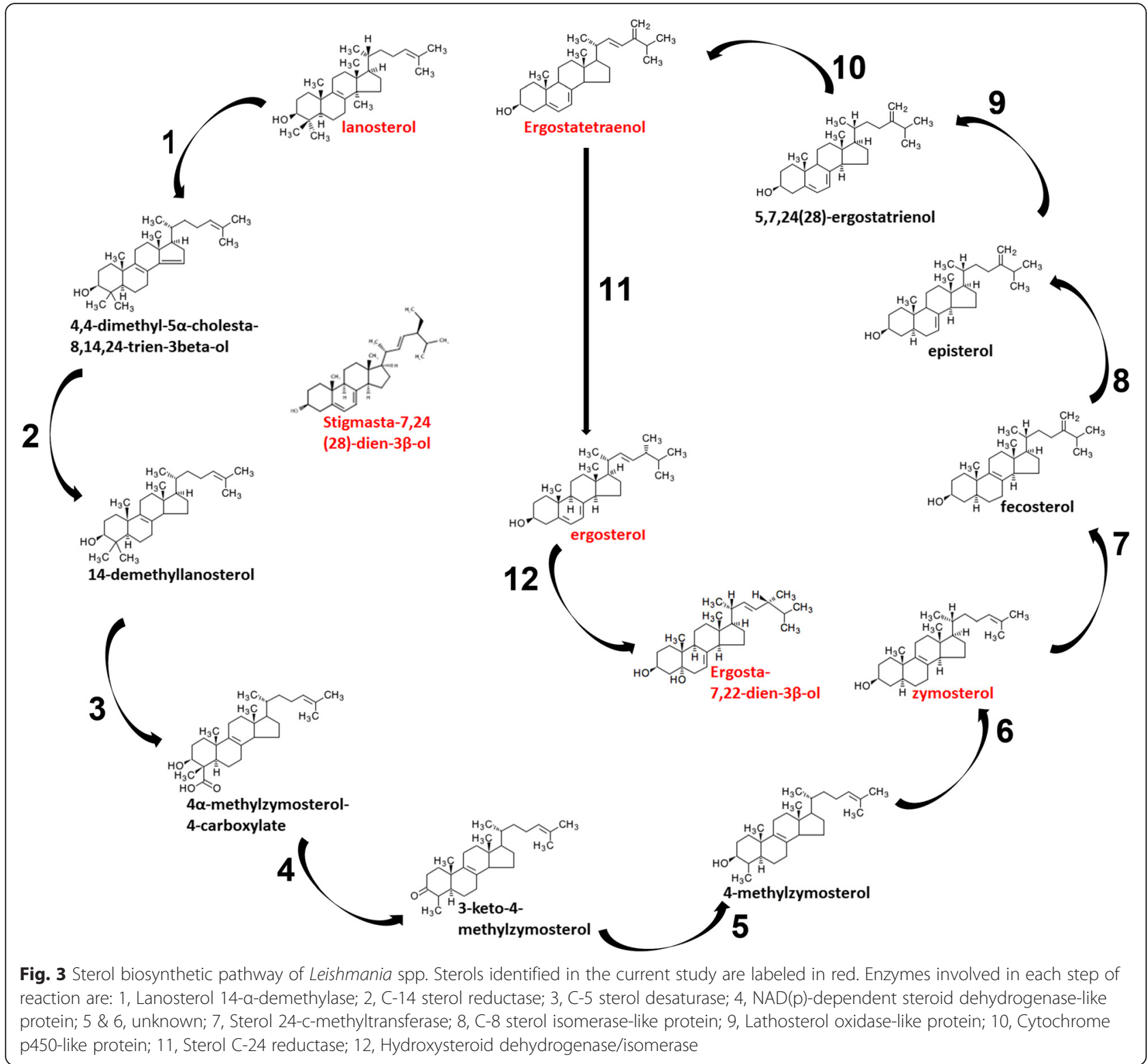

CoA reductase (HMG-CoA reductase). The first committed step toward phytosterol biosynthesis is SMT1 (C-24 sterol methyl transferase), and the subsequent action of SMT2, which catalyzes a methyl transfer to methylene lophenol, directs the product toward sitosterol and stigmasterol biosynthesis. The annotated genomes of both $L$. infantum and $T$. brucei include genes predicted to encode a HMG-CoA reductase (LinJ.30.3230 and Tb927.6.4540). Two genes for a sterol 24-C methyl transferase are found in the L. infantum JPCM5 genome: LinJ.36.2510 and LinJ.36.2520, as is a sterol C-24 reductase (LinJ.33.0730) and several other (C-8, C-14) sterol reductases. The T. brucei genome also encodes two sterol 24-C-methyl transferases (TB11.v5.0496, Tb927.10.6950) and only one annotated (C-14) sterol reductase. Whether the enzymes of $L$. infantum participate in stigmastanol synthesis, and whether the additional sterol reductase genes present in $L$. infantum over $T$. brucei are influential in the differential recognition of stigmastanol only in L. infantum, cannot be determined without further experimental evidence. ([37], trytrypdb.org).

This would not be the first discovery of a pathway in Leishmania spp. that is more closely related to fungal metabolic pathways than those of Trypanosoma brucei. Indeed, T. brucei take in iron primarily through a transferrin receptor, whereas the Leishmania species use a two-step surface ferric iron reductase $\left(\mathrm{Fe}^{3+} \rightarrow \mathrm{Fe}^{2+}\right) / \mathrm{fer}-$ rous iron transferase [38-40].

Calculations of absolute amounts of each sterol allowed us to assess (1) whether the total sterol content 
is associated with parasite virulence, and (2) whether adsorbed cholesterol inhibits ergosterol synthesis. Relevant to the first question, the total amount of sterols in $1 \times 10^{7}$ stationary-phase promastigotes was $1,766.0 \mathrm{ng}$, roughly double the amount in purified metacyclic promastigotes from the same culture (810.2 ng, Table 3). The difference was mainly accounted for by a reduction in ergosterol I and II, with a lowering of adsorbed cholesterol as a second contributory change. L. infantum stationary-phase promastigotes contain $41.4 \%$ metacyclic cells [3], so the remaining non-metacyclic promastigotes must have accounted for the differences. This difference may be partially explained by a smaller body size of the metacyclic promastigotes observed in both flow cytometry [41, 42] and microscopy [3, 41, 43-46], although this cannot be the full explanation since there were proportional differences between sterols in the populations, and because most sterols are located at the cellular membrane $[47,48]$. Nevertheless, the significant difference in proportions suggest differences in ergosterol synthesis may contribute. Metabolic changes during metacyclogenesis could in part be favored by the absence of purine, or by an acidic environment [49-51]. It is possible that other environmental conditions such as environmental cholesterol/sterol concentrations also favor metacyclogenesis.

Cholesterol and ergosterol are integral components of plasma membrane micro-domains called lipid rafts, also known as detergent-resistant membrane (DRMs) [52]. $\mathrm{M} \beta C D$ specifically chelates and depletes sterols from lipid rafts. In the current study we confirmed our earlier report that $M \beta C D$ treatment of metacyclic promastigotes reduced the total amount of cellular sterols by two thirds. We previously reported that lipid-raft depleted metacyclic promastigotes exhibit increased sensitivity to complement medicated lysis in vitro and reduced infectivity for mice in vivo [8].

Leishmania spp. are inoculated by the sand fly into mammalian tissue in a pool of blood, a relatively cholesterol rich environment. Our observations raise the possibility that suspension in such an environment might alter the membrane sterol content of the inoculated parasite. Host cholesterol levels affect the outcomes of Leishmania spp. infections. It has been reported that high circulating lipid levels offer protection against $L$. donovani infection in a mouse model where mice were either provided a high-cholesterol (atherogenic) diet or underwent statin treatment [53]. Similarly, patients with active VL had a lower level of total cholesterols during acute disease than four months after VL resolution in the same patients, and lower cholesterol than healthy volunteer controls [54]. Whether transient exposure to this environment at the initial stage of infection affects the virulence of promastigotes is a matter of speculation.
From the current work we can conclude that the sterol content of Leishmania promastigotes is dynamic and responds not only to environmental conditions, but also the developmental changes that occur during metacyclogenesis. These changes seem to be a result of both adsorption of cholesterol from the environment, and likely also ergosterol synthesis by the Leishmania spp. parasites. The interaction between sterol content and parasite virulence is a yet evolving story.

\section{Conclusions}

Leishmaniasis causes 20,000 annual deaths and is a threat to $5 \%$ of the populations of four continents worldwide. Its causative pathogens include more than 20 species of protozoan parasites in the genus of Leishmania, and there is an association between parasite species and clinical outcomes. The pathogenic mechanisms used by the parasite are incompletely understood, and a better understanding could speed both vaccine development and new drug discovery. We hypothesized that dynamic changes in sterol composition during parasite development contribute to development of virulent forms of the parasite that are the most infectious for mammalian hosts such as humans. Qualitative and quantitative measures of sterols using gas chromatograph-mass spectrometry enabled us to contrast sterols in infectious versus non-infectious parasite forms. Our data clearly showed a unique profile of sterols in the infectious parasites, and revealed that external environment is a major determinate of cellular sterol content. These discoveries warrant further investigation of roles of sterols in Leishmania spp. pathogenesis.

\section{Additional files}

\section{Additional file 1: Table S1. Effect of different culture media on absolute sterol content of stationary-phase promastigotes of a virulent strain compared to an avirulent strain of Leishmania infantum ${ }^{+}$. (DOCX 13 kb) \\ Additional file 2: Table S2. Homologous genes of Leishmania spp. sterol biosynthetic pathway (SBP). Trypanosoma cruzi SBP genes were used as gene ID during database search except those marked with an "*" in which keyword search was used. Blank cells indicate data not available. (XLSX $17 \mathrm{~kb}$ ) \\ Additional file 3: Figure S1. Identification of stigmasta-7,24(28)- dien-3 $\beta$-ol by GC-MS. A: GC-MS peaks of the compound at Relative retention time (RRt) of 1.229. B: Peaks of CAS \#481-19-6, stigmasta- 7,24(28)-dien-3ß-ol. C: Comparison of the peaks in $A$ and $B$ and relative amount for each peak is shown. (PPTX $100 \mathrm{~kb}$ )}

\footnotetext{
Abbreviations

CL: cutaneous leishmaniasis; DRM: detergent resistant membranes; FCS: fetal calf serum; GC-MS: gas chromatograph-mass spectrometry; KEGG: Kyoto Encyclopedia of Genes and Genomes; M $\beta C D$ : methyl- $\beta$-cyclodextrin; SFM: serum free medium; RRt: relative retention time; VL: visceral leishmaniasis.
}

Competing interests

The authors declare that they have no competing interests. 


\section{Authors' contributions}

CY conceived of the study, and participated in its design, and performed all experiments and helped to draft the manuscript. MEW conceived of the study, and helped to draft the manuscript. All authors read and approved the final manuscript.

\section{Acknowledgment}

The authors are very grateful to Dr. Lynn M. Teesch of High Resolution Mass Spectrometry Facility, University of lowa for her help in analyzing sterols by GC-MS. The cost of publication is paid by an intramural grant of One Health Center for Zoonoses and Tropical Veterinary Medicine of Ross University School of Veterinary Medicine. The work was funded in part by $\mathrm{NIH}$ grants R01 Al076233 and R01 Al045540, and Merit Review grants 5I01BX001983 and 2101BX000536 from the Department of Veterans' Affairs.

\section{Author details}

'Department of Biomedical Sciences and One Health Center for Zoonoses and Tropical Veterinary Medicine, Ross University School of Veterinary Medicine, P.O. Box 334, Basseterre, St. Kitts, West Indies. ${ }^{2}$ Departments of Internal Medicine, Microbiology and Epidemiology, University of lowa, lowa City, IA USA. ' Iowa City VA Medical Center, lowa City, IA USA.

Received: 4 December 2015 Accepted: 23 March 2016 Published online: 12 April 2016

\section{References}

1. Desjeux P. Leishmaniasis: current situation and new perspectives. Comp Immunol Microbiol Infect Dis. 2004;27(5):305-18.

2. Bates PA. Transmission of Leishmania metacyclic promastigotes by phlebotomine sand flies. Int J Parasitol. 2007;37(10):1097-106.

3. Yao C, Chen Y, Sudan B, Donelson JE, Wilson ME. Leishmania chagasi: Homogenous metacyclic promastigotes isolated by buoyant density are highly virulent in a mouse model. Exp Parasitol. 2008;118:129-33.

4. Roberts CW, McLeod R, Rice DW, Ginger M, Chance ML, Goad LJ. Fatty acid and sterol metabolism: potential antimicrobial targets in apicomplexan and trypanosomatid parasitic protozoa. Mol Biochem Parasitol. 2003:126(2):129-42.

5. Goad LJ, Holz JGG, Beach DH. Sterols of Leishmania species, implications for biosynthesis. Molecular \& Biochemical Parasitology. 1984;10:161-70.

6. Denny PW, Field MC, Smith DF. GPI-anchored proteins and glycoconjugates segregate into lipid rafts in Kinetoplastida. FEBS Lett. 2001;491(1-2):148-53.

7. Denny PW, Goulding D, Ferguson MA, Smith DF. Sphingolipid-free Leishmania are defective in membrane trafficking, differentiation and infectivity. Mol Microbiol. 2004;52(2):313-27.

8. Yao C, Gaur Dixit U, Barker JH, Teesch LM, Love-Homan L, Donelson JE, Wilson ME. Attenuation of Leishmania infantum chagasi metacyclic promastigotes by sterol depletion. Infect Immun. 2013;81(7):2507-17.

9. Mishra BB, Kale RR, Singh RK, Tiwari VK. Alkaloids: Future prospective to combat leishmaniasis. Fitoterapia. 2009;80(2):81-90.

10. Sundar S. Drug resistance in Indian visceral leishmaniasis. Trop Med Int Health. 2001;6(11):849-54.

11. Durand R, Paul M, Pratlong F, Rivollet D, Dubreuil-Lemaire ML, Houin R, Astier A, Deniau M. Leishmania infantum: lack of parasite resistance to amphotericin B in a clinically resistant visceral leishmaniasis. Antimicrob Agents Chemother. 1998;42(8):2141-3.

12. Ellis D. Amphotericin B: spectrum and resistance. J Antimicrob Chemother. 2002;49 Suppl 1:7-10.

13. Xu W, Hsu FF, Baykal E, Huang J, Zhang K. Sterol biosynthesis is required for heat resistance but not extracellular survival in Leishmania. PLoS Pathog. 2014;10(10):e1004427.

14. Roberts SC, Wilson ME, Donelson JE. Developmentally regulated expression of a novel 59-kDa product of the major surface protease (Msp or gp63) gene family of Leishmania chagasi. J Biol Chem. 1995;270(15):8884-92.

15. Yao C, Donelson JE, Wilson ME. Internal and surface-localized MSP of Leishmania and their differential release from promastigotes. Eukaryot Cell. 2007;6(10):1905-12.

16. Cauchetier E, Loiseau PM, Lehman J, Rivollet D, Fleury J, Astier A, Deniau M, Paul M. Characterisation of atovaquone resistance in Leishmania infantum promastigotes. Int J Parasitol. 2002;32(8):1043-51.

17. Cosentino RO, Aguero F. Genetic profiling of the isoprenoid and stero biosynthesis pathway genes of Trypanosoma cruzi. PLoS One. 2014;9(5):e96762.
18. Berriman M, Ghedin E, Hertz-Fowler C, Blandin G, Renauld H, Bartholomeu DC, Lennard NJ, Caler E, Hamlin NE, Haas B Lennard NJ, Caler E, Hamlin NE, Haas B et al. The genome of the African trypanosome Trypanosoma brucei. Science. 2005;309(5733):416-22.

19. El-Sayed NM, Myler PJ, Bartholomeu DC, Nilsson D, Aggarwal G, Tran AN, Ghedin E, Worthey EA, Delcher AL, Blandin G et al. The genome sequence of Trypanosoma cruzi, etiologic agent of Chagas disease. Science. 2005;309(5733):409-15.

20. El-Sayed NM, Myler PJ, Blandin G, Berriman M, Crabtree J, Aggarwal G, Caler E, Renauld H, Worthey EA, Hertz-Fowler C et al. Comparative genomics of trypanosomatid parasitic protozoa. Science. 2005;309(5733):404-9.

21. Ivens AC, Peacock CS, Worthey EA, Murphy L, Aggarwal G, Berriman M, Sisk E, Rajandream MA, Adlem E, Aert R et al. The genome of the kinetoplastid parasite, Leishmania major. Science. 2005;309(5733):436-42.

22. McCall L-I, El Aroussi A, Choi JY, Vieira DF, De Muylder G, Johnston JB, Chen S, Kellar D, Siqueira-Neto JL, Roush WR et al. Targeting ergosterol biosynthesis in Leishmania donovani: essentiality of sterol 14alphademethylase. PLoS Negl Tropic Dis. 2015;9(3):e0003588.

23. Cota GF, de Sousa MR, de Mendonca AL, Patrocinio A, Assuncao LS, de Faria SR, Rabello A. Leishmania-HIV co-infection: clinical presentation and outcomes in an urban area in Brazil. PLoS Negl Tropic Dis. 2014;8(4):e2816.

24. Rakotomanga M, Blanc S, Gaudin K, Chaminade P, Loiseau PM. Miltefosine affects lipid metabolism in Leishmania donovani promastigotes. Antimicrob Agents Chemother. 2007;51(4):1425-30.

25. Lorente SO, Rodrigues JC, Jimenez Jimenez C, Joyce-Menekse M, Rodrigues C, Croft SL, Yardley V, de Luca-Fradley K, Ruiz-Perez LM, Urbina J et al. Novel azasterols as potential agents for treatment of leishmaniasis and trypanosomiasis. Antimicrob Agents Chemother. 2004;48(8):2937-50.

26. Torres-Santos EC, Sampaio-Santos MI, Buckner FS, Yokoyama K, Gelb M, Urbina JA, Urbina JA, Rossi-Bergmann B. Altered sterol profile induced in Leishmania amazonensis by a natural dihydroxymethoxylated chalcone. J Antimicrob Chemother. 2009;63(3):469-72.

27. Rakotomanga M, Saint-Pierre-Chazalet M, Loiseau PM. Alteration of fatty acid and sterol metabolism in miltefosine-resistant Leishmania donovani promastigotes and consequences for drug-membrane interactions. Antimicrob Agents Chemother. 2005;49(7):2677-86.

28. de Souza W, Rodrigues JC. Sterol biosynthesis pathway as target for antitrypanosomatid drugs. Interdiscip Perspect Infect Dis. 2009;2009:642502.

29. Nes Craigen R, Singha Ujjal K, Liu J, Ganapathy K, Villalta F, Waterman Michael R, Lepesheva Galina I, Chaudhuri M, Nes WD. Novel sterol metabolic network of Trypanosoma brucei procyclic and bloodstream forms. Biochem J. 2012;443(1):267-77.

30. Oehlschlager AC, Angus RH, Pierce AM, Pierce Jr HD, Srinivasan R. Azasterol inhibition of delta 24-sterol methyltransferase in Saccharomyces cerevisiae. Biochemistry. 1984;23(16):3582-9.

31. Osumi T, Taketani S, Katsuki H, Kuhara T, Matsumoto I. Ergosterol biosynthesis in yeast. Pathways in the late stages and their variation under various conditions. J Biochem. 1978;83(3):681-91.

32. Niedermeyer TH, Lindequist U, Mentel R, Gordes D, Schmidt E, Thurow K, Lalk M. Antiviral terpenoid constituents of Ganoderma pfeifferi. J Nat Prod. 2005;68(12):1728-31.

33. Giner JL, Zhao $\mathrm{H}$. Detailed sterol compositions of two pathogenic rust fungi. Lipids. 2004;39(8):763-7.

34. Mahto KK, Singh A, Khandelwal NK, Bhardwaj N, Jha J, Prasad R. An assessment of growth media enrichment on lipid metabolome and the concurrent phenotypic properties of Candida albicans. PLoS One. 2014;9(11):1-20.

35. Acuna-Johnson AP, Oehlschlager AC, Pierce AM, Pierce Jr HD, Czyzewska EK Stereochemistry of yeast $\triangle 24$-sterol methyl transferase. Bioorg Med Chem. 1997;5(5):821-32.

36. Chapuis L, Malosse C, Corio-Costet MF. Sterol composition of the woody plant pathogenic fungus Eutypa lata. Phytochemistry. 1996;42(6):1599-601.

37. Neelakandan AK, Chamala S, Valliyodan B, Nes WD, Nguyen HT. Metabolic engineering of soybean affords improved phytosterol seed traits. Plant Biotechnol J. 2012;10(1):12-9.

38. Flannery AR, Renberg RL, Andrews NW. Pathways of iron acquisition and utilization in Leishmania. Curr Opin Microbiol. 2013;16(6):716-21.

39. Wilson ME, Lewis TS, Miller MA, McCormick ML, Britigan BE. Leishmania chagasi: uptake of iron bound to lactoferrin or transferrin requires an iron reductase. Exp Parasitol. 2002;100(3):196-207.

40. Wilson ME, Vorhies RW, Andersen KA, Britigan BE. Acquisition of iron from transferrin and lactoferrin by the protozoan Leishmania chagasi. Infect Immun. 1994;62(8):3262-9. 
41. da Silva IA, Morato Cl, Quixabeira VBL, Pereira LIA, Dorta ML, de Oliveira MAP, et al. In Vitro Metacyclogenesis of Leishmania (Viannia) braziliensis and Leishmania (Leishmania) amazonensis clinical field isolates, as evaluated by morphology, complement resistance, and infectivity to human macrophages. Bio Med Res Int. 2015;2015:15.

42. Saraiva EM, Pinto-da-Silva LH, Wanderley JL, Bonomo AC, Barcinski MA Moreira ME. Flow cytometric assessment of Leishmania spp. metacyclic differentiation: Validation by morphological features and specific markers. Exp Parasitol. 2005;110(1):39-47.

43. Bandyopadhyay P, Ghosh DK, De A, Ghosh KN, Chaudhuri PP, Das P, et al. Metacyclogenesis of Leishmania spp.: species-specific in vitro transformation complement resistance, and cell surface carbohydrate and protein profiles. J Parasitol. 1991;77(3):411-6.

44. Howard MK, Sayers G, Miles MA. Leishmania donovani metacyclic promastigotes: transformation in vitro, lectin agglutination, complement resistance, and infectivity. Exp Parasitol. 1987;64(2):147-56.

45. Nieves E, Pimenta PF. Development of Leishmania (Viannia) braziliensis and Leishmania (Leishmania) amazonensis in the sand fly Lutzomyia migonei (Diptera: Psychodidae). J Med Entomol. 2000;37(1):134-40.

46. Pinto-da-Silva LH, Camurate M, Costa KA, Oliveira SM, da Cunha-e-Silva NL, Saraiva EM. Leishmania (Viannia) braziliensis metacyclic promastigotes purified using Bauhinia purpurea lectin are complement resistant and highly infective for macrophages in vitro and hamsters in vivo. Int J Parasitol. 2002;32(11):1371-7.

47. Sturley SL. Conservation of eukaryotic sterol homeostasis: new insights from studies in budding yeast. Biochim Biophys Acta. 2000;1529(1-3):155-63.

48. Jacquier N, Schneiter R. Mechanisms of sterol uptake and transport in yeast. J Steroid Biochem Mol Biol. 2012;129(1-2):70-8.

49. Bates PA, Tetley L. Leishmania mexicana: induction of metacyclogenesis by cultivation of promastigotes at acidic pH. Exp Parasitol. 1993;76(4):412-23.

50. Serafim TD, Fiqueiredo AB, Costa PAC, Marques-da-Silva EA, Gonçalves R, de Moura SAL, et al. Leishmania metacyclogenesis is promoted in the absence of purines. PLoS Negl Trop Dis. 2012:6(9):e1833.

51. Zakai HA, Chance ML, Bates PA. In vitro stimulation of metacyclogenesis in Leishmania braziliensis, L. donovani, L. major and L. mexicana. Parasitology. 1998;116(Pt 4):305-9.

52. Goluszko P, Nowicki B. Membrane cholesterol: a crucial molecule affecting interactions of microbial pathogens with mammalian cells. Infect Immun. 2005:73(12):7791-6.

53. Ghosh J, Das S, Guha R, Ghosh D, Naskar K, Das A, et al. Hyperlipidemia offers protection against Leishmania donovani infection: role of membrane cholesterol. J Lipid Res. 2012:53(12):2560-72.

54. Liberopoulos EN, Apostolou F, Gazi IF, Kostara C, Bairaktari ET, Tselepis AD, et al. Visceral leishmaniasis is associated with marked changes in serum lipid profile. Eur J Clin Invest. 2014;44(8):719-27.

\section{Submit your next manuscript to BioMed Central and we will help you at every step:}

- We accept pre-submission inquiries

- Our selector tool helps you to find the most relevant journal

- We provide round the clock customer support

- Convenient online submission

- Thorough peer review

- Inclusion in PubMed and all major indexing services

- Maximum visibility for your research

Submit your manuscript at www.biomedcentral.com/submit

) Biomed Central 